\title{
Retraction Note: A multi-agent architectural solution for coherent distributed reconfigurations of function blocks
}

Mohamed Khalgui • Olfa Mosbahi •

Hans-Michael Hanisch • Zhiwu Li

Published online: 4 July 2013

(C) Springer Science+Business Media New York 2013

Retraction to: J Intell Manuf (2012) 23:2531-2549

DOI 10.1007/s10845-011-0556-y

This paper has been withdrawn due to similarities to the previously published paper "Reconfiguration of Distributed Embedded-Control Systems" by M. Khalgui, O. Mosbahi, Zhiwu Li, H.-M. Hanisch, IEEE/ASME Transactions on Mechatronics, Vol. 16, No. 4, pp. 684-694, 2011.

The online version of the original article can be found under doi:10.1007/s10845-011-0556-y.

M. Khalgui $(\varangle) \cdot$ O. Mosbahi $\cdot$ Z. Li

Xidian University, Xi' an, China

e-mail: khalgui.mohamed@gmail.com

H.-M. Hanisch

Martin Luther University, Halle Saale, Germany 